\title{
Determination of bubble point pressure of two live oils with injected nitrogen by quartz crystal resonator
}

\author{
Ezequiel Orlandi ${ }^{\mathrm{a}}$, Jean-Luc Daridon ${ }^{\mathrm{b}}$, and Hervé Carrier ${ }^{\mathrm{c}}$ \\ Laboratoire des Fluides Complexes et leurs Réservoirs, UMR 5150, Université de Pau et
} des Pays de l'Adour, BP 1155, 64013 Pau Cedex, France

\begin{abstract}
An experimental investigation of bubble pressure determination of two live oils with injected nitrogen is presented in this work. Conventional bubble point measurement is done with the aid of a constant expansion test. In such method the bubble point is characterized by an inflection in the PV curve as the oil becomes saturated. For some oils the PV curve is smoothed and it becomes difficult or impossible to accurately determine the bubble point. This work demonstrates that in such cases, the use of quartz crystal resonator technique makes it possible. This technique is successfully applied to determine the bubble point pressure of two live oils in which nitrogen is injected up to $40 \mathrm{~mol} \%$.
\end{abstract}

\section{Introduction}

Bubble point pressure of crude oils is a crucial parameter in field development and operations. When live oil sampling is possible, bubble point pressure determination can be performed by a constant mass expansion (CME) in a PVT laboratory [1]. Bubble point is routinely determined from a sharp change in the PV curve due to change in compressibility once the oil becomes saturated. For volatile, near critical oils and oils that could have undergone secondary recovery processes the PV curve is smoothened and the accuracy of bubble point determination is jeopardized. For this reason numerical and experimental methods are constantly improved in order to accomplish a better crude oil characterization.

Our group recently published a detailed study on the bubble point pressure determination in bottomhole oils by using quartz crystal resonator technique [2]. It was shown that bubble point pressure of live oils can be estimated with a very good accuracy of $0.05 \mathrm{MPa}$ by monitoring resonant properties (frequency and energy dissipation) of a quartz resonator immersed in a live oil.

\footnotetext{
a e-mail: ezequiel.orlandidesouzafreire@univ-pau.fr

b e-mail: jean-luc.daridon@univ-pau.fr

c e-mail: herve.carrier@univ-pau.fr
} 


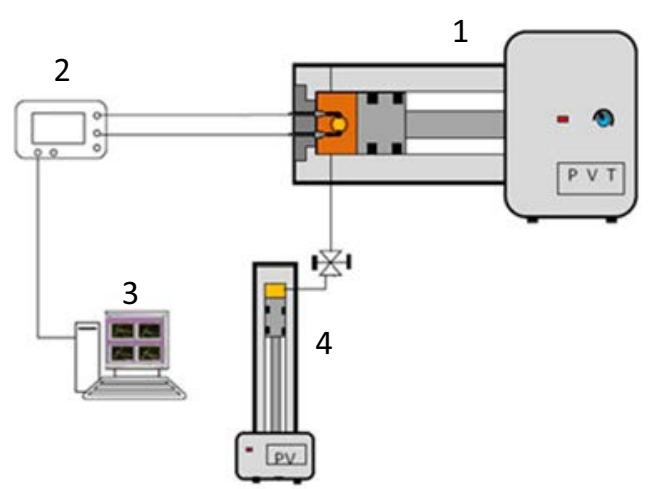

Fig. 1. Schematics of the PVT cell equipped with a quartz crystal resonator (1); analyzer (2) and computer (3) to record frequency and bandwidth data and to control the PVT cell pressure or volume; (4) a syringe pump utilized to inject nitrogen into the PVT cell.

Dissolved nitrogen is normally found in crude oil in small amount [3]. Higher proportions can be a result of nitrogen miscible flooding. Nitrogen flooding is generally done to maintain reservoir pressure above saturation conditions and to enhance oil recovery $[4,5]$. Such kind of secondary recovery technique is applicable mostly for light oils and reservoirs with a depth capable of supporting pressures above the nitrogen minimum miscible pressure without causing formation damages.

Nitrogen and other flooding gases change crude oil bubble point pressure [6,7]. Nitrogen causes a huge increase in crude oil bubble point whereas other flooding gases such as $\mathrm{CO}_{2}$ may cause a decrease of bubble conditions. Changes in bubble pressures related to gas injection must be determined with accuracy in order to design flooding process. However, the addition of nitrogen in live crude oil changes their compressibilities and makes smaller the discontinuity in the derivative of the volume versus pressure at the bubble point. In such cases the bubble point may not be determined with accuracy from usual PV method and more sensitive methods have to be applied. In this context, the aim of this work was to investigate the phase behavior of live oil with addition of nitrogen by using a quartz crystal resonator. To this end, measurements were carried out in two different crude oils with a nitrogen content ranging from 0 to $40 \mathrm{~mol} \%$.

\section{Experimental section}

\subsection{Apparatus and live oils}

The phase behavior of live oil + nitrogen system was measured up to $100 \mathrm{MPa}$ using synthetic method with an indirect detection based on the monitoring of the resonant properties of a quartz crystal resonator. The apparatus, which has been described previously in detail [2] and shown in Figure 1, is made up of an automatic mobile piston cell closed at one end by a plug holding an acoustic wave sensor. It consists in a highly polished AT-cut beveled quartz disk with a fundamental frequency of $3 \mathrm{MHz}$ and a blank diameter of $13.6 \mathrm{~mm}$. Two electrodes were deposited on its faces on the central plano-plano portion by vacuum evaporation of an adhesive layer of titanium of $10 \mathrm{~nm}$ thickness followed by a $100 \mathrm{~nm}$ thick layer of gold. The external parts of the pins are connected to a network analyzer thanks to coaxial cable with SMA connectors. The cell is placed horizontally as well as the quartz sensor so as it probes only the liquid phase whatever the existence or nonexistence of a gas cap. The 
cylinder is fitted with two valves, one near to the end plug serving and inlet valve, and the other in the middle of the cylinder serves as discharging valve.

The live oil, received in a high pressure container, is brought to reservoir conditions (i.e. reservoir temperature and pressure) and is then transferred in small increments to the pre-evacuated PVT cell at the same temperature as the live oil container.

The oil that is transferred to the cell is maintained at reservoir pressure and temperature during 12 hours under stirring by a magnetic stirrer and three magnetic stir balls placed inside the cell. The volume occupied by the oil sample in the cell is measured to evaluate the mass of fluid introduced. The real amount of live oil introduced is determined according to PVT data provided by the oil company i.e. densities values and molar mass. This knowledge is crucial for the rest of the measurements since mixture composition is calculated according to that initial input. An accurate calibration of the dead volume of the cell is necessary. It has been done at the temperatures of interest in the pressure range 1-100 MPa (taking into account stainless steel dilatation with pressure and temperature) by introducing different mass of toluene, used as a reference compound for which densities are known with high accuracy (data are from NIST).

After the equilibrium time a stepwise depressurization at constant temperature is performed along with the recording of frequency and bandwidth data by the quartz crystal resonator to check if the bubble point pressure matches the bubble point pressure from the reservoir. This guarantees the integrity of the sample after the transfer process. The system is re-pressurized and the injection of nitrogen is then performed with the aid of a syringe pump with temperature control. The pressure in the syringe pump is brought to a higher pressure than the cell and the pump is set to maintain such a pressure. The nitrogen density at pressure and temperature of the syringe pump is used to calculate the volume of nitrogen which is needed to be injected in the cell to attain the desired molar composition of the system. The initial pressure and volume at the determined temperature at the syringe pump is noted. The valve that connects the syringe pump to the cell is then opened and the syringe pump starts to inject nitrogen in the cell. Once the desired nitrogen volume is injected the valve is closed and the final volume of the syringe pump is noted when the pressure stabilizes. The experimental quantity of injected nitrogen is then calculated having the experimental injected volume and the density of nitrogen at the temperature and pressure on the syringe pump.

When nitrogen is injected the system is tested to check if the mixture is monophasic. The test consist of a gradual depressurization following the energy dissipation profile. If there is no sudden change in the slope of the dissipation profile the system can be considered monophasic. The inverse process can also be applied. By an increase of pressure, if the dissipation profile has no change in its slope the system is considered monophasic. The effect of a monophasic system in energy dissipation is regular and is dictated by the change in viscosity and density of the solution as pressure increases or decreases.

During experiments, the system is constantly mixed by using magnetic stirrers. Temperatures for the experiments of crude oil 1 and 2 were set to their reservoir conditions (347 and $368 \mathrm{~K}$ respectively). Depressurizations were normally done at a constant expansion rate of $0.3 \mathrm{MPa}$ per minute. Compositional analysis of live oil 1 and 2 are shown in Table 1.

\subsection{Quartz crystal resonator technique}

Quartz crystals are piezoelectric materials which when cut on the proper crystallographic plane are able to oscillate in shear mode when an alternating current is applied 
Table 1. Molar compositional analysis and respective molecular weights from the compounds present in North Sea live oil 1 and 2.

\begin{tabular}{lccccc}
\hline \multirow{2}{*}{ Component } & \multicolumn{2}{c}{ Live oil 1} & & \multicolumn{2}{c}{ Live oil 2} \\
\cline { 2 - 3 } \cline { 5 - 6 } & mol $\%$ & $\mathrm{Mw}$ & & mol $\%$ & $\mathrm{Mw}$ \\
\hline $\mathrm{N}_{2}$ & 1.0 & 28.0 & & 0.6 & 28.0 \\
\hline $\mathrm{CO}_{2}$ & 0.1 & 44.0 & & 0.1 & 44.0 \\
\hline $\mathrm{C}_{1}$ & 19.6 & 16.0 & & 19.7 & 16.0 \\
\hline $\mathrm{C}_{2}$ & 5.6 & 30.1 & & 5.1 & 30.1 \\
\hline $\mathrm{C}_{3}$ & 8.5 & 44.1 & & 6.2 & 44.1 \\
\hline $\mathrm{i}-\mathrm{C}_{4}$ & 1.4 & 58.1 & & 1.4 & 58.1 \\
\hline $\mathrm{n}-\mathrm{C}_{4}$ & 5.6 & 58.1 & & 3.3 & 58.1 \\
\hline $\mathrm{i}-\mathrm{C}_{5}$ & 2.1 & 72.2 & & 1.4 & 72.2 \\
\hline $\mathrm{n}-\mathrm{C}_{5}$ & 2.9 & 72.2 & & 1.9 & 72.2 \\
\hline Fraction $\mathrm{C}_{6}$ & 3.8 & 84.9 & & 2.6 & 84.2 \\
\hline Fraction $\mathrm{C}_{7+}$ & 49.4 & 268.3 & 57.7 & 224.0 \\
\hline
\end{tabular}

across its faces. Its connection to a network analyzer makes it possible the two main parameters of the vibrating quartz, its resonant frequency $(f)$ and the half-band-halfwidth $(\Gamma)$ to be recorded. The half-band-half-width of the conductance spectra is related to energy dissipation $(\Delta D)$ as expressed by equation (1)

$$
\Delta D=\frac{2 \Delta \Gamma}{f} .
$$

Quartz crystal resonator technique was first used as a means of micro-weighting or thickness evaluation of thin and elastic films by Sauerbray [8]. Sauerbray showed that a frequency decrease on the vibrating system is directly proportional to the thickness increase of a thin and elastic overlayer. Later Nomura and Kanazawa [9] developed the experimental and theoretical basis for the use of quartz crystal immersed in Newtonian liquid. They found that the frequency was directly proportional to the square root of the density viscosity product of the fluid. Muramatsu et al. [10] showed that the energy dissipation $(\Delta D)$ of a quartz immersed in a Newtonian fluid is also directly to the square root of the density viscosity product of the fluid. Equation (2) brings the energy dissipation as expressed by the half-band-half-width of a quartz with both faces immersed in a Newtonian liquid.

$$
\Delta \Gamma=f_{0}^{3 / 2}\left(\frac{\eta_{l} \rho_{l}}{\pi \mu_{q} \rho_{q}}\right)^{1 / 2}
$$

where $\Delta \Gamma$ is the change in bandwidth compared to the bandwidth of the vibrating quartz under vacuum conditions and $\eta_{l}, \rho_{l}, \mu_{q}, \rho_{q}$ stand for liquid viscosity, liquid density, quarts shear modulus and quartz density respectively. $f_{0}$ is the fundamental resonant frequency as measured under vacuum.

In a system without or with negligible adsorption or deposition on the surface of the quartz, bandwidth response is only dependent on the density and viscosity of the bulk fluid. Therefore, bandwidth of a quartz crystal resonator can be used to sense any changes in density and or viscosity of a fluid in contact with quartz faces. This characteristics of the quartz makes the bandwidth a good parameter for probing bubble conditions. The use of resonant frequency is also possible to detect phase changes, but its sensitivity to adsorbed material and also to pressure changes lessens the accuracy of bubble point determination.

Quartz crystal resonator technique can be applicable with the fundamental and overtones resonating modes. Former studies had demonstrated [2] the higher accuracy 


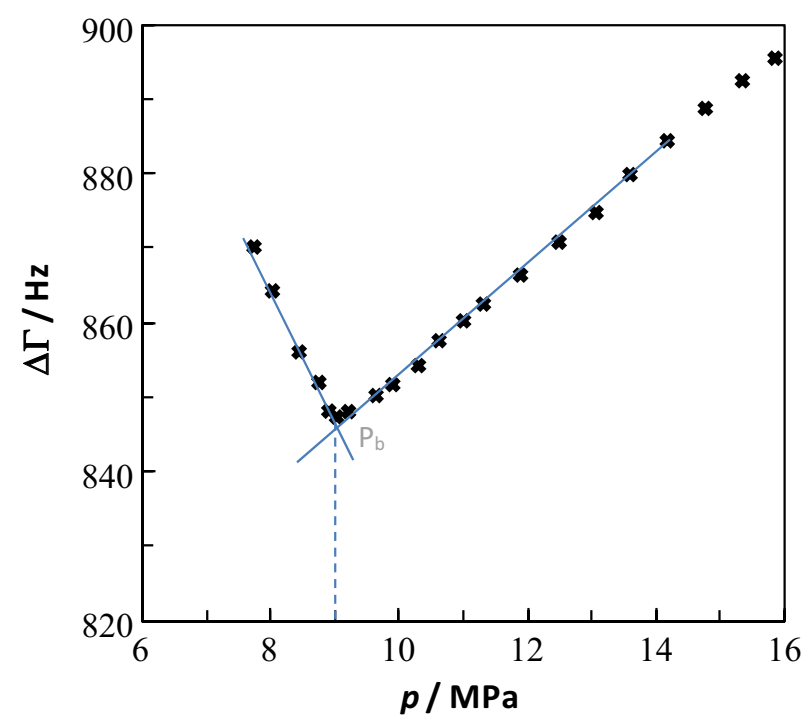

Fig. 2. Change in dissipation (half-band-half-width) measured by a Quartz Crystal Resonator immersed in live oil 1 during a constant mass expansion experiment at $T=367.65 \mathrm{~K}$. $\mathbf{X}$ : experiment; : linear interpolation of either side of the corner point.

on the conductance spectra acquisition of the third overtone mode when compared to the fundamental and higher harmonics. The fundamental overtone is essentially influenced by the quartz dimensions and higher overtones are more prone to be disturbed by inharmonics signals that may interact with harmonic peaks when the quartz resonator is immersed in viscous fluids [11].

\section{Results and discussions}

The constant mass expansion test with the use of quartz crystal resonator for live oil 1 is shown in Figure 2. Above the saturation pressure bandwidth data monotonically decreases as pressure is reduced due to an overall decrease in density and viscosity of the fluid. Once the bubble point is attained there is a sharp minimum in bandwidth data. In such a point as gas is released from the live oil the increase in density and viscosity of the media drives the augment in bandwidth. The bubble point is then determined as the intersection of two straight lines fitted on both sides of the inflection region. On the under-saturated region the decompression leads to the decrease of density and viscosity of the system, however, on the saturated region two effects are occurring. The sole decompression leads to a further decrease on density and viscosity on the liquid phase which is contra balanced by the increase in density and viscosity due to gas liberation.

The determination of the bubble point for live oil 1 without injected nitrogen can be easily obtained from a constant mass expansion test. However with the addition of nitrogen there is a continuous smoothening of the compressibility of the fluid on the under-saturated and saturated regions. PV data obtained from CME tests on live oil 1 with different nitrogen molar fraction are shown in Figure 3. For nitrogen molar fraction of $40 \%$ the effect of the nitrogen saturation on the proximities of the bubble point can be observed (Fig. 3). The high nitrogen content smoothens the PV curve making the bubble point determination very inaccurate. 


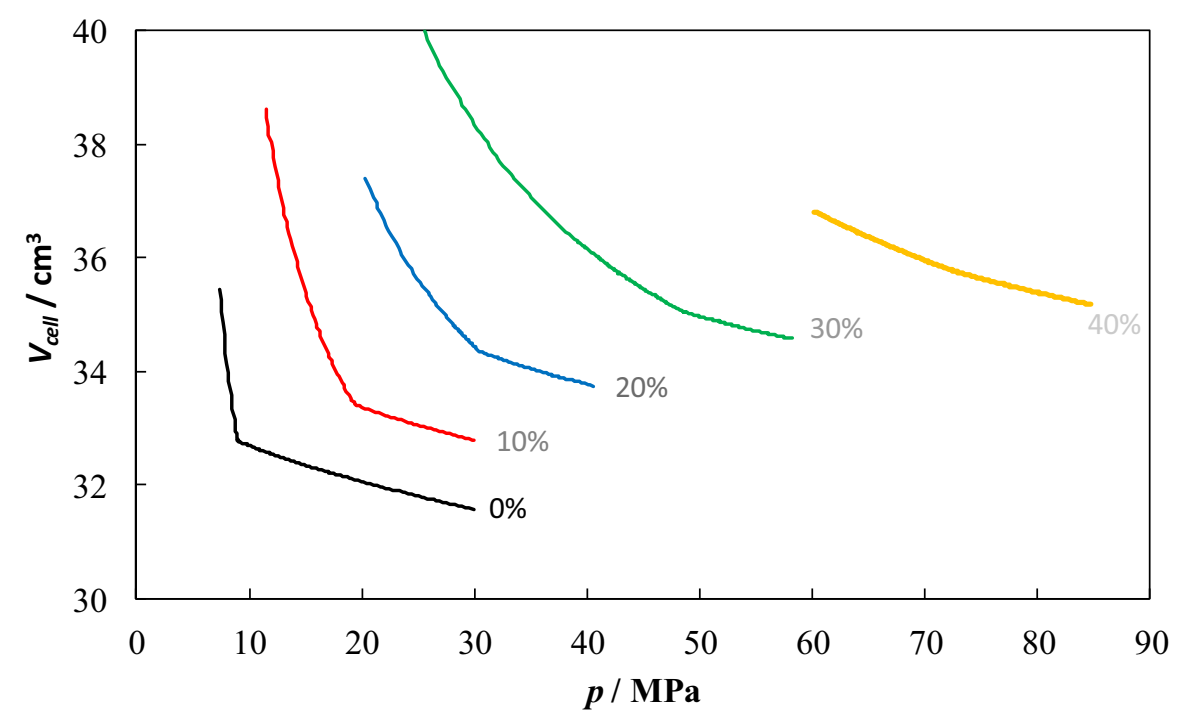

Fig. 3. Pressure - Volume curve measured in various mixtures of $\mathrm{N}_{2}+$ live oil 1 during a constant mass expansion experiment at $T=367.65 \mathrm{~K}$. $: 30 \%$; $: 40 \%$ of $\mathrm{N}_{2}$ in $\mathrm{mol} \%$.

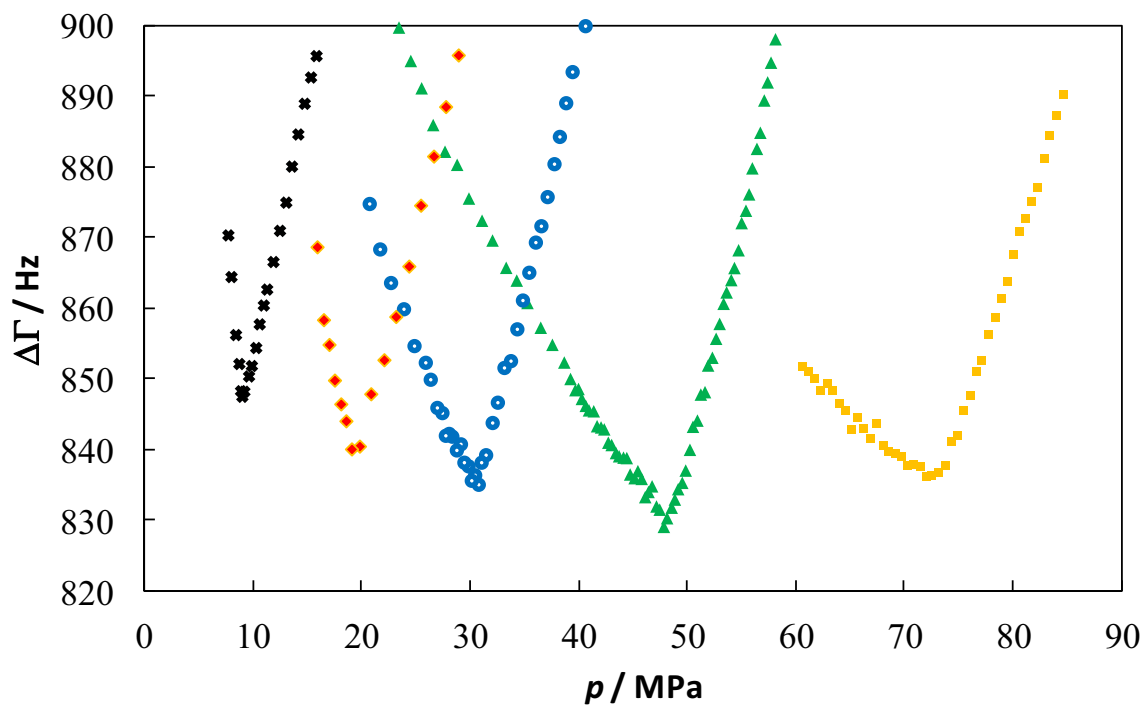

Fig. 4. Change in dissipation (half-band-half-width) measured by a Quartz Crystal Resonator immersed in various mixtures of $\mathrm{N}_{2}+$ live oil 1 during a constant mass expansion experiment at $T=367.65 \mathrm{~K} . \mathbf{X}: 0 \% ; \bullet: 10 \% ; \mathbf{O}: 20 \% ; \Delta: 30 \% ; \square: 40 \%$ of $\mathrm{N}_{2}$ in $\mathrm{mol} \%$.

The use of quartz crystal and analysis of the bandwidth profile during decompression highlights bubble point pressure for all the studied nitrogen molar fractions as shown in Figure 4. The similar profile of the curves inclination in the under saturated regions puts in evidence the sole effect of density and viscosity reduction which is occurring in such a macroscopically monophasic fluid. 


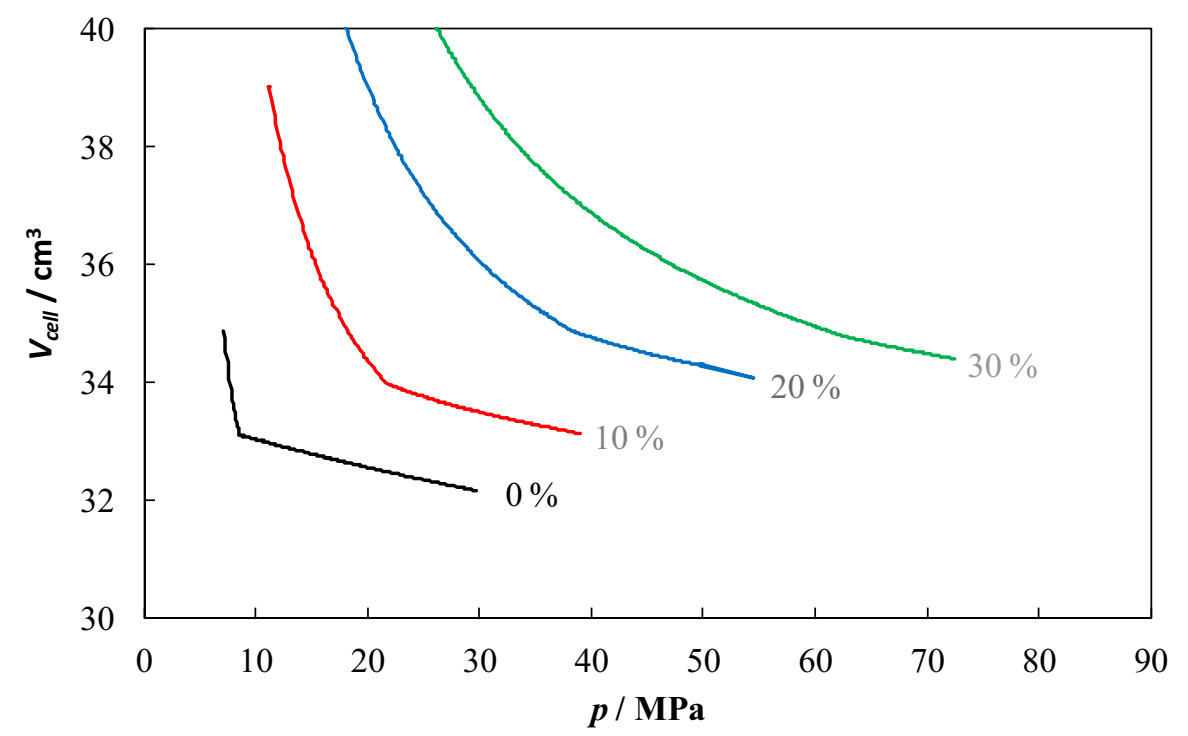

Fig. 5. Pressure - Volume curve measured in various mixtures (in mol $\%$ of $\mathrm{N}_{2}$ ) of $\mathrm{N}_{2}+$ live oil 2 during a constant mass expansion experiment at $T=347.87 \mathrm{~K}$ $: 0 \%$ : $10 \%$; : $20 \%$; $: 30 \%$; of $\mathrm{N}_{2}$ in $\mathrm{mol} \%$.

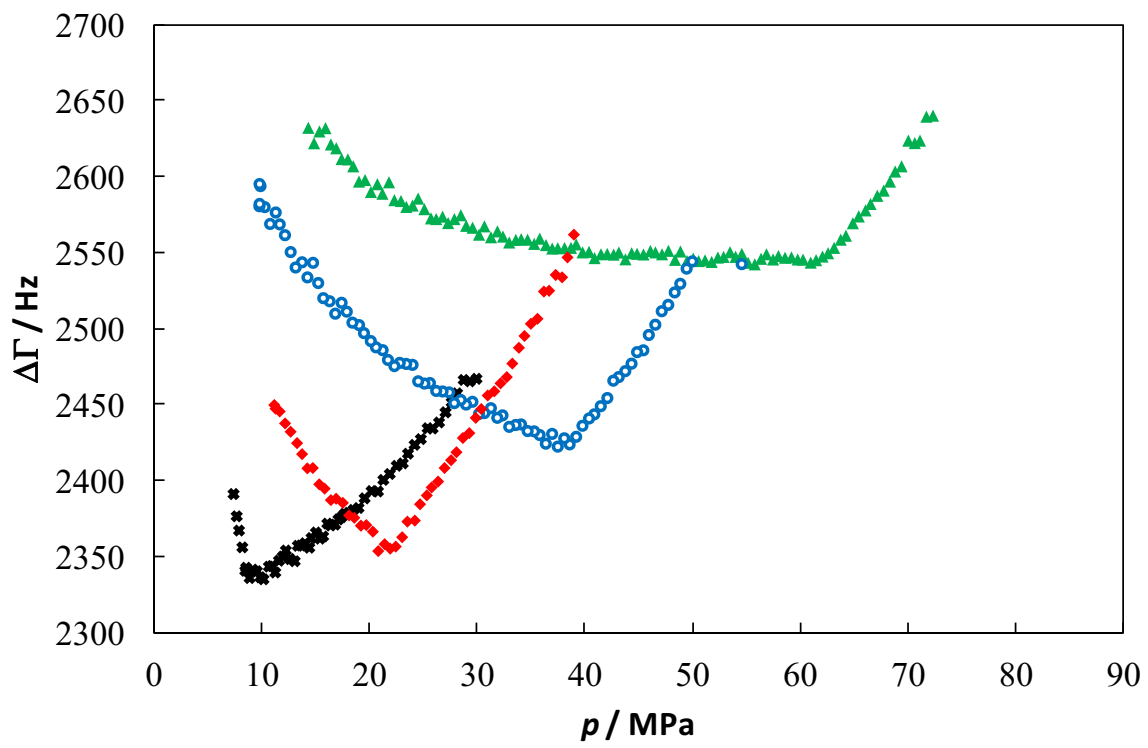

Fig. 6. Change in dissipation (half-band-half-width) measured by a Quartz Crystal Resonator immersed in various mixtures of $\mathrm{N}_{2}+$ live oil 2 during a constant mass expansion experiment at $T=347.87 \mathrm{~K} . \mathbf{X}: 0 \%$; $\diamond: 10 \% ; \mathbf{O}: 20 \% ; \Delta: 30 \%$; of $\mathrm{N}_{2}$ in $\operatorname{mol} \%$.

The obtained results for the constant mass expansion test for live oil 2 is shown in Figure 5. Figure 6 brings the results obtained on the bandwidth analysis for the constant mass expansion of crude oil 2.

The bubble points as they were determined by the bandwidth data and PV method are summarized in Table 2 for live oil 1 and 2 with the different nitrogen molar fractions. The bubble point for the live oil 2 with $40 \mathrm{~mol} \%$ nitrogen is not shown as 
Table 2. Bubble point pressure measured in Live oil + injected nitrogen pseudo binary systems.

\begin{tabular}{|c|c|c|c|c|c|c|c|}
\hline $\begin{array}{l}x_{N_{2}} \\
(\mathrm{~mol} \%)\end{array}$ & $T / \mathrm{K}$ & $\begin{array}{l}P / \mathrm{MPa} \\
\text { Quartz }\end{array}$ & $\begin{array}{c}P / \mathrm{MPa} \\
\text { PV method }\end{array}$ & $\begin{array}{c}x_{N_{2}} \\
(\mathrm{~mol} \%) \\
\end{array}$ & $T / \mathrm{K}$ & $\begin{array}{l}P / \mathrm{MPa} \\
\text { Quartz }\end{array}$ & $\begin{array}{c}P / \mathrm{MPa} \\
\mathrm{PV} \text { method }\end{array}$ \\
\hline \multicolumn{5}{|c|}{$\mathrm{N}_{2}+$ Live oil 1} & \multicolumn{3}{|c|}{$\mathrm{N}_{2}+$ Live oil 2} \\
\hline 0 & 367.7 & 9.04 & 8.99 & 0 & 347.9 & 8.53 & 8.45 \\
\hline 9.95 & 368.1 & 19.52 & 19.47 & 11.68 & 348.1 & 21.87 & 21.78 \\
\hline 19.27 & 368.1 & 30.48 & 30.46 & 21.13 & 348.2 & 38.31 & 38.22 \\
\hline 29.41 & 368.4 & 48.64 & 49.15 & 30.33 & 348.1 & 62.00 & 62.37 \\
\hline 39.25 & 368.4 & 72.95 & 72.90 & 40.36 & 348.3 & $>100$ & $>100$ \\
\hline
\end{tabular}

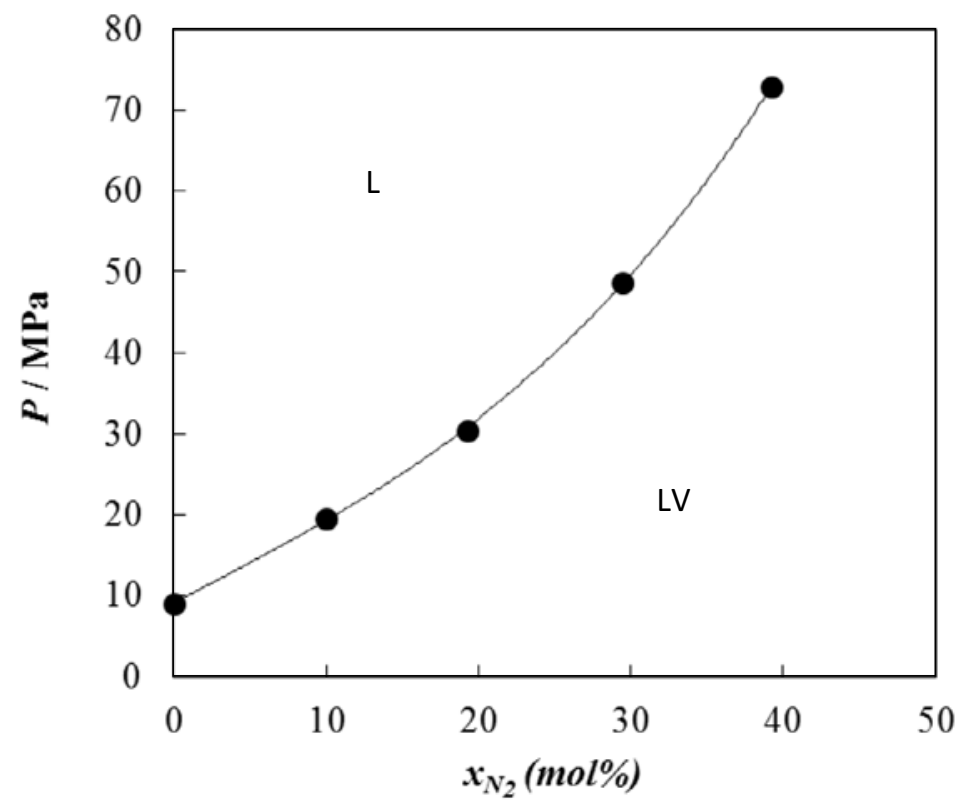

Fig. 7. Bubble point pressure as a function of nitrogen content in $\mathrm{N}_{2}+$ Live oil 1 pseudo binary system.

it was higher than $100 \mathrm{MPa}$ which is close to the highest pressure that the PVT cell was designed to support.

The pseudo phase diagram of the systems were then plotted and are shown in Figures 7 and 8 .

\section{Conclusion}

In continuation of the former developed work [2] the present study confirms the successful applicability of quartz crystal resonator technique to determine bubble point in live crude oil samples with injected nitrogen. The analysis of the bandwidth is preferred as it is independent on pressure and less sensitive to adsorbed material. The third harmonic is the less affected by quartz dimensions and inharmonic modes. Further studies with this technique will be performed with the aim of evaluating the rheology of the systems before and after nitrogen injection. It is worthy to highlight 


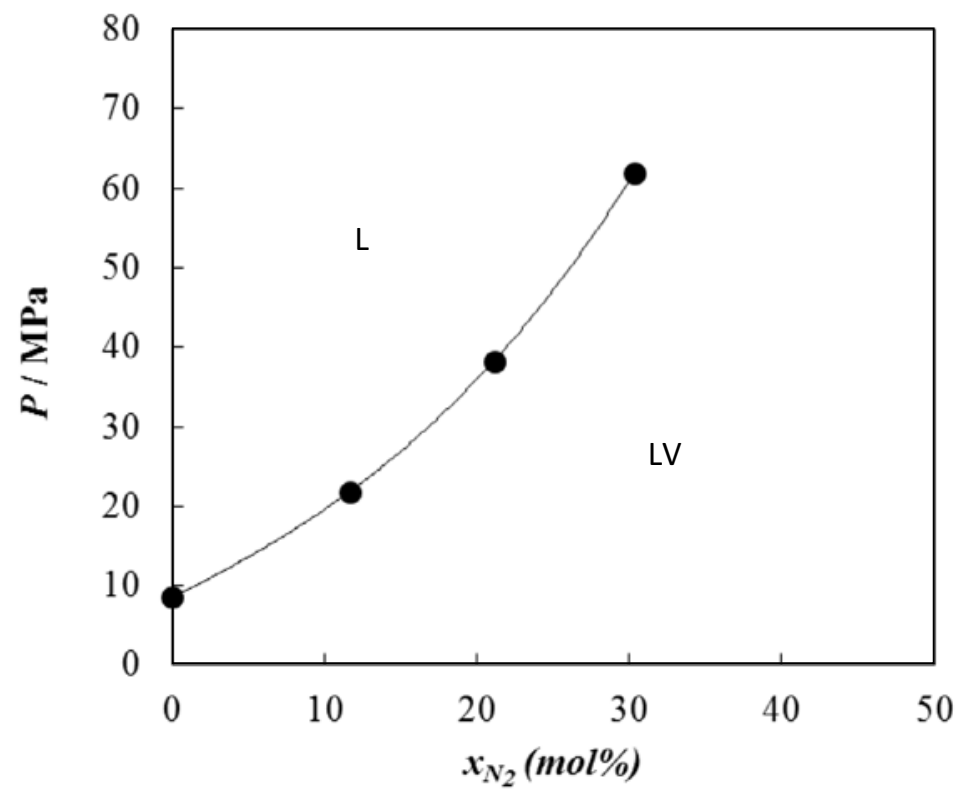

Fig. 8. Bubble point pressure as a function of nitrogen content in $\mathrm{N}_{2}+$ Live oil 2 pseudo binary system.

that the use of quartz crystal resonator technique can also be applied to evaluate the influence of other gases as methane or carbon dioxide on live oil bubble point.

This work was carried out as a part of the Joint Industrial Programme (JIP) Asphaltenes consortium "Improved Mechanisms of Asphaltene Deposition and Precipitation to Minimize Irregularities in Production and Transport" sponsored by the following industrial partners AkzoNobel, BP, Canada Natural Resources, Nalco Champion, Petrobras, Statoil and Total as well as by the Norwegian Research Council (234112/E30).

\section{References}

1. L.P. Dake, Fundamentals of Reservoir Engineering, 1st edn. (Elsevier, Amsterdam, 1978)

2. J.L. Daridon, E. Orlandi, H. Carrier, Fluid Phase Equilib. 427, 152 (2016)

3. C.H. Whitson, M.R. Brule, Phase Behavior (SPE, Texas, 2010)

4. J.G. Speight, The Chemistry and Technology of Petroleum, 5th edn. (CRC Press, 2014)

5. J.P. Clancy, R.E. Gilchrist, D.E. Kroll, Nitrogen for the enhanced revovery of oil and gas, SPE-9912-M, 1981

6. J.L. Vogel, L. Yarborough, The effect of nitrogen on the phase behavior and physical properties of reservoir fluids, SPE-8815, 1980

7. O. Glas $\varnothing$, Generalized Pressure-Volume-Temperature Correlations, SPE-8016-PA, 1980

8. G. Sauerbrey, Z. Phys. 155, 206 (1959)

9. K.K. Kanazawa, J.G. Gordon, Anal. Chem. 57, 1770 (1985)

10. H. Muramatsu, E. Tamiya, I. Karube, Anal. Chem. 60, 2142 (1988)

11. M. Cassiede, J.L. Daridon, J.H. Paillol, J. Pauly, Sens. Actuators 167, 317 (2011) 